\title{
Synthesis of New Macrocyclic Chiral Manganese(III) Schiff Bases as Catalysts for Asymmetric Epoxidation
}

\author{
Alexandre Martinez, Catherine Hemmert,* Christophe Loup, Guillaume Barré and Bernard \\ Meunier* \\ Laboratoire de Chimie de Coordination du CNRS, 205 route de narbonne, 31077 Toulouse \\ cedex 4, France \\ hemmert@lcc-toulouse.fr ; bmeunier@lcc-toulouse.fr
}




\section{Table of Contents}

General experimental methods $\mathrm{S} 2$

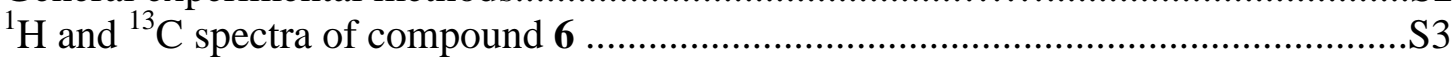

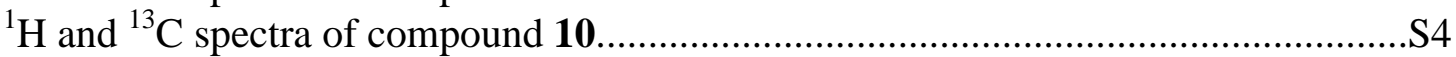

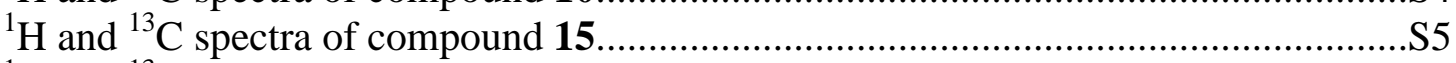

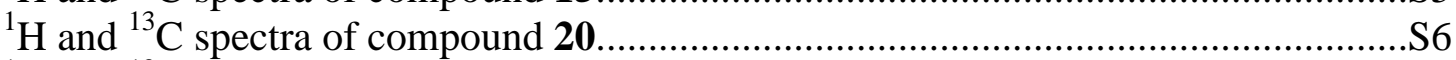

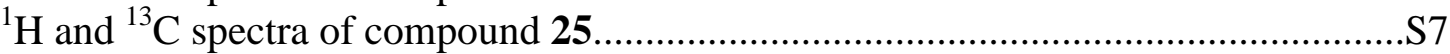

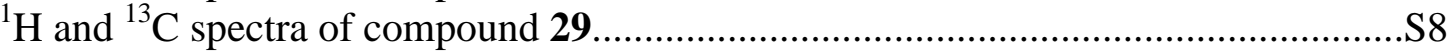

General experimental methods. ${ }^{1} \mathrm{H}$ NMR were recorded at $250 \mathrm{MHz}$ in $\mathrm{CDCl}_{3}$ as internal reference. ${ }^{13} \mathrm{C}$ NMR spectra were recorded at $62.8 \mathrm{MHz}$. Mass spectrometry analyses were performed using $\mathrm{EI}, \mathrm{FAB}^{+}$or ES techniques in the positive mode. UV-visible spectra were obtained on a diode array spectrophotometer. IR spectra were recorded on a FT-IR spectrophotometer. Optical rotations were measured with a polarimeter. Gas chromatography (GC) analyses were performed on a chromatograph equipped with a flame ionization detector and a chiral capillary column (betadex $120,30 \mathrm{~m} \times 0.25 \mathrm{~mm}, 0.25 \mu \mathrm{m}$ film) and coupled to an integrator. The injector temperature was $240{ }^{\circ} \mathrm{C}$ and the column temperature $140{ }^{\circ} \mathrm{C}$, with a column pressure of 10 psi. 1,4-Dibromobenzene was used as internal standard for the GC analyses.2,6-Dibromo-4-tert-butylphenol was prepared as described in the literature. ${ }^{[1]} \mathrm{Di}$ and tri-ethylene glycol were di-tosylated according literature procedure. ${ }^{[2]}$ 5-allyloxy-1,3benzenedimethyl ditosylate was synthetized in three steps from 5-hydroxyisophthalic acid. ${ }^{[3]}$ Other commercially available reagents and solvents were purchased from standard chemical suppliers and used without further purification.

[1] Chan, K. S.; Xu, J. X.; Lam, F. J. Org. Chem. 1996, 61, 8414-8418.

[2] Cornforth, J. W.; Morgan, F. D.; Potts, K. T.; Rees, R. T. W. Tetrahedron 1973, 29, 1659-1667.

[3] Bradshaw J. S., Huszthy, P.; Wang, T.; Zhu, C.; Nazarenko, A. Y.; Izatt, R. M. Supramolecular Chem. 1993, 1, 267-273. 


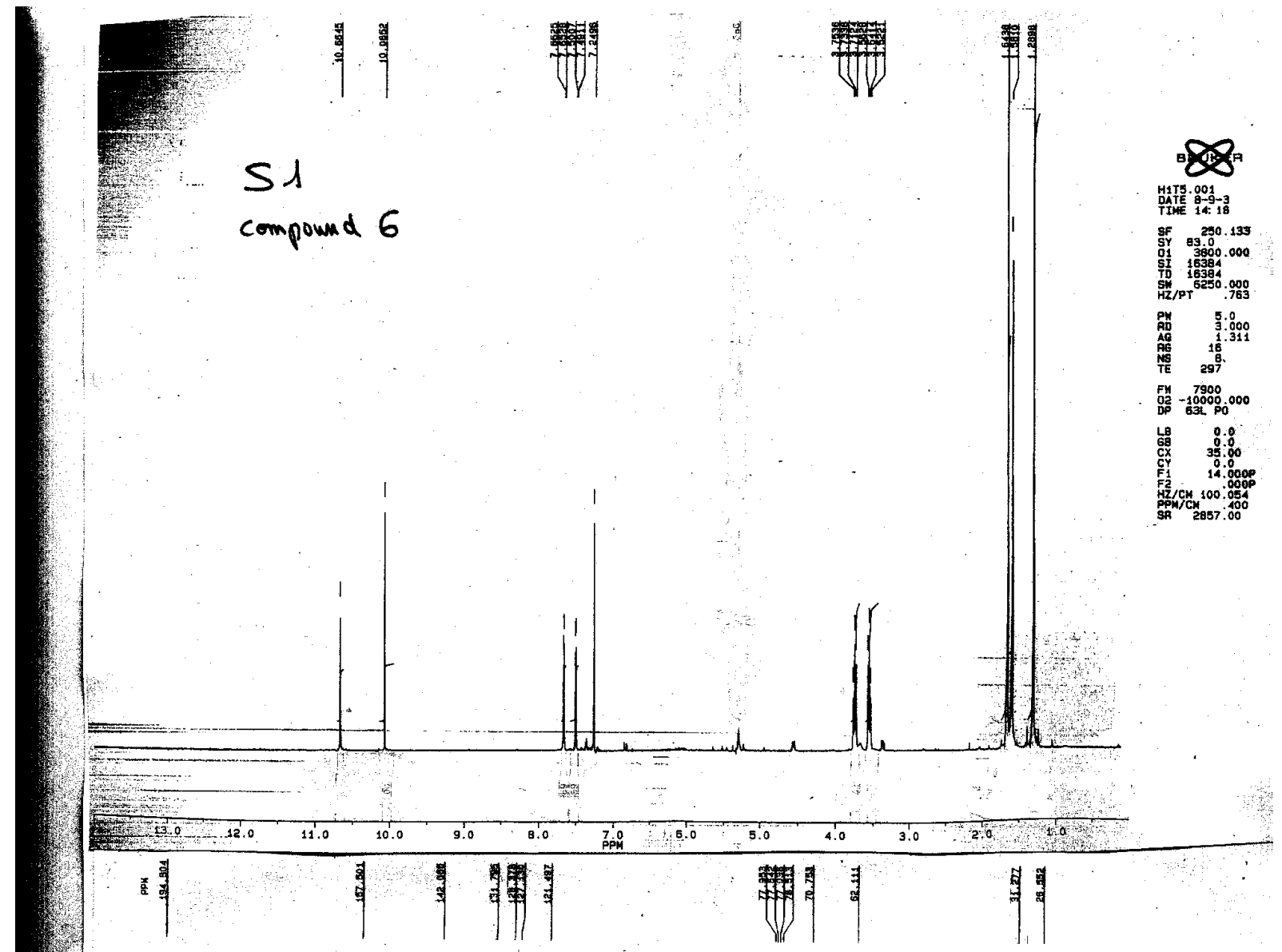

S2

compound 6

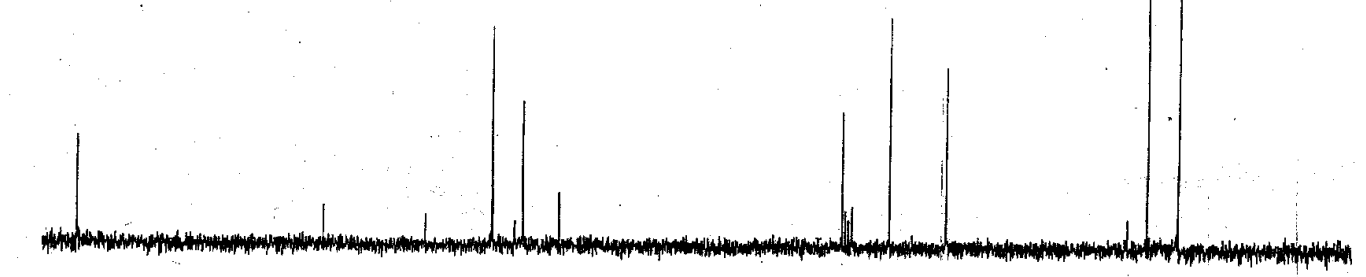

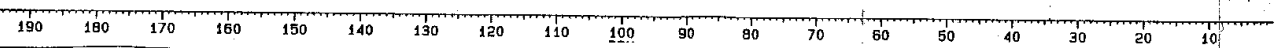




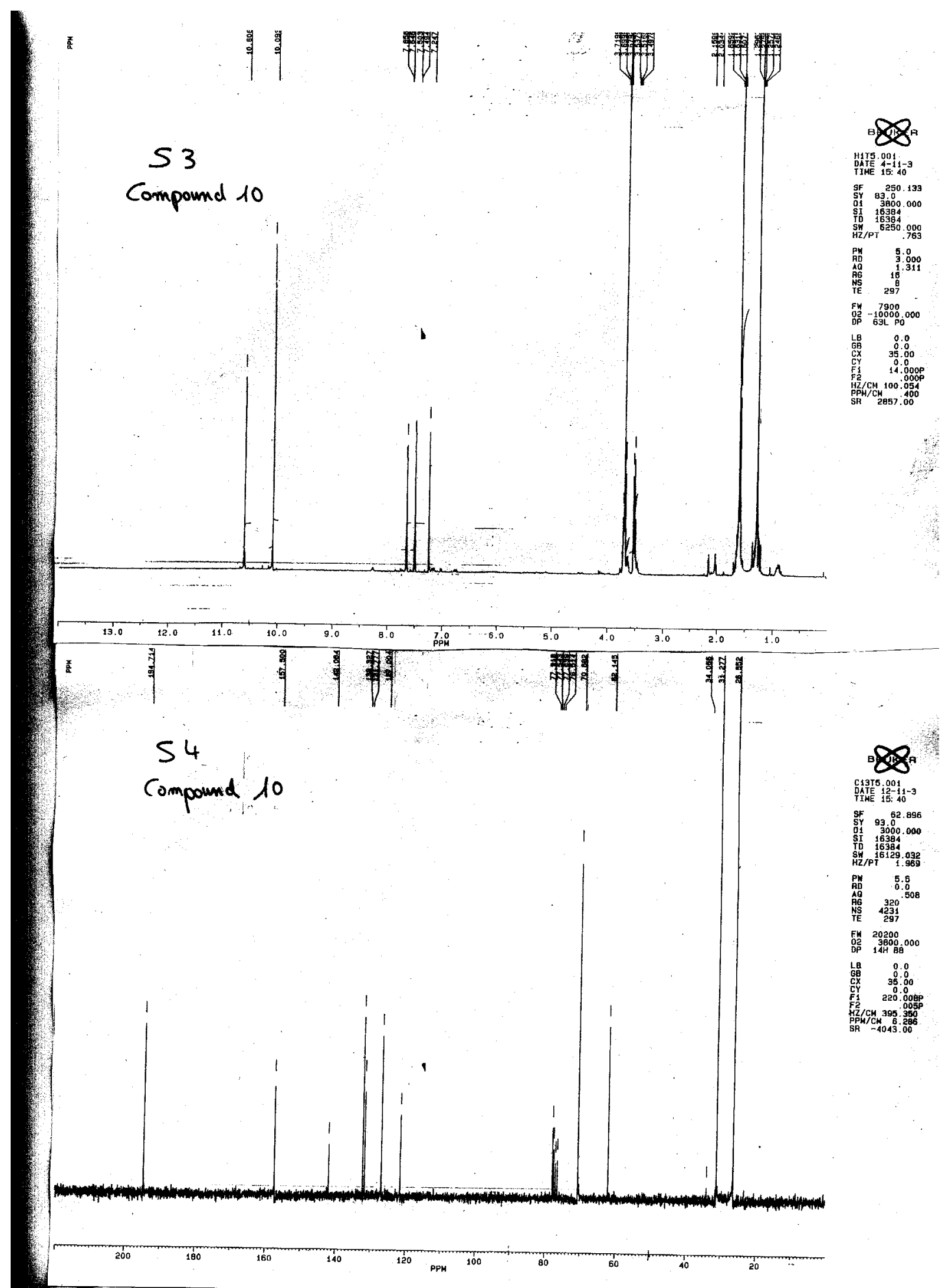




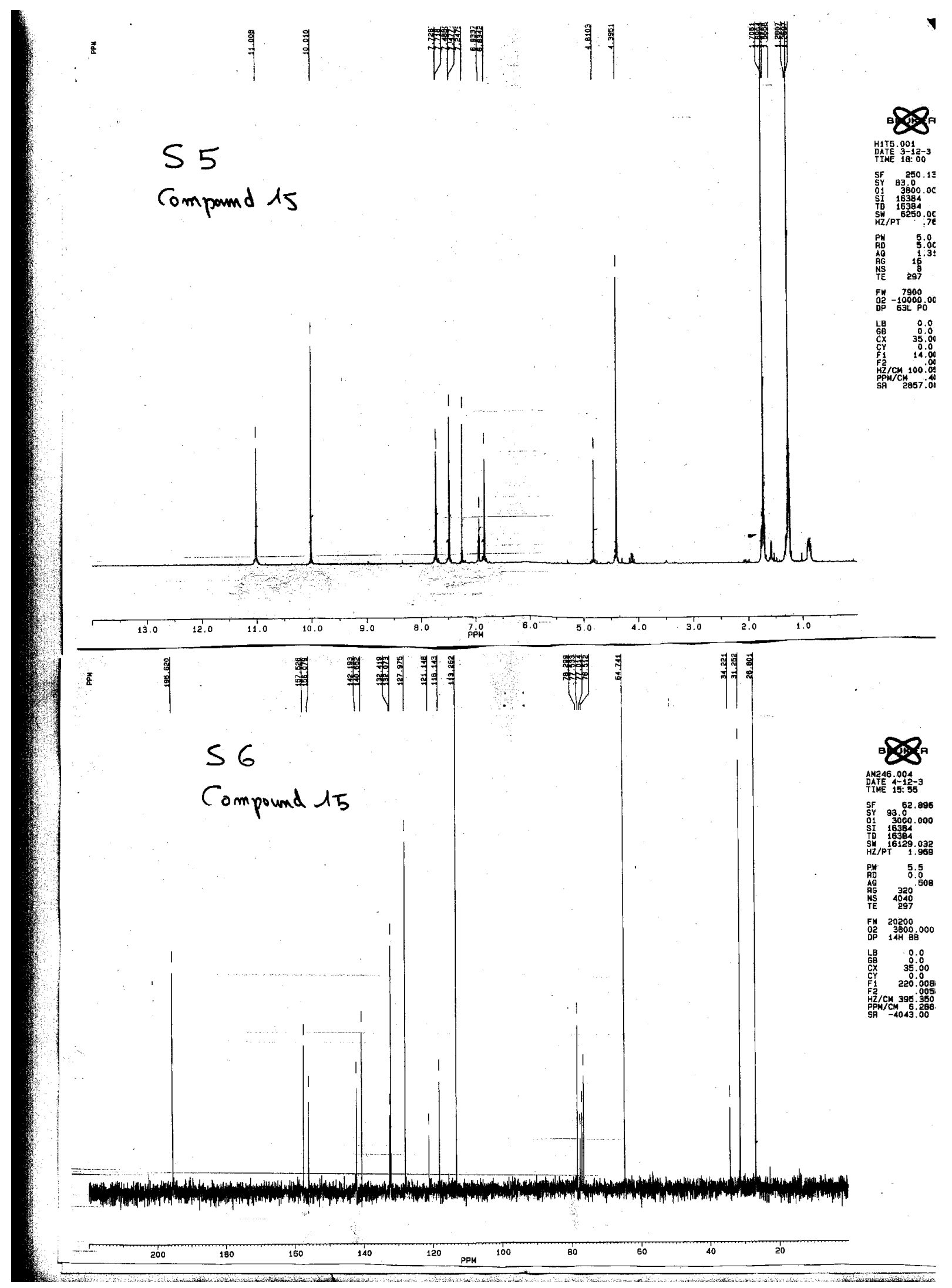



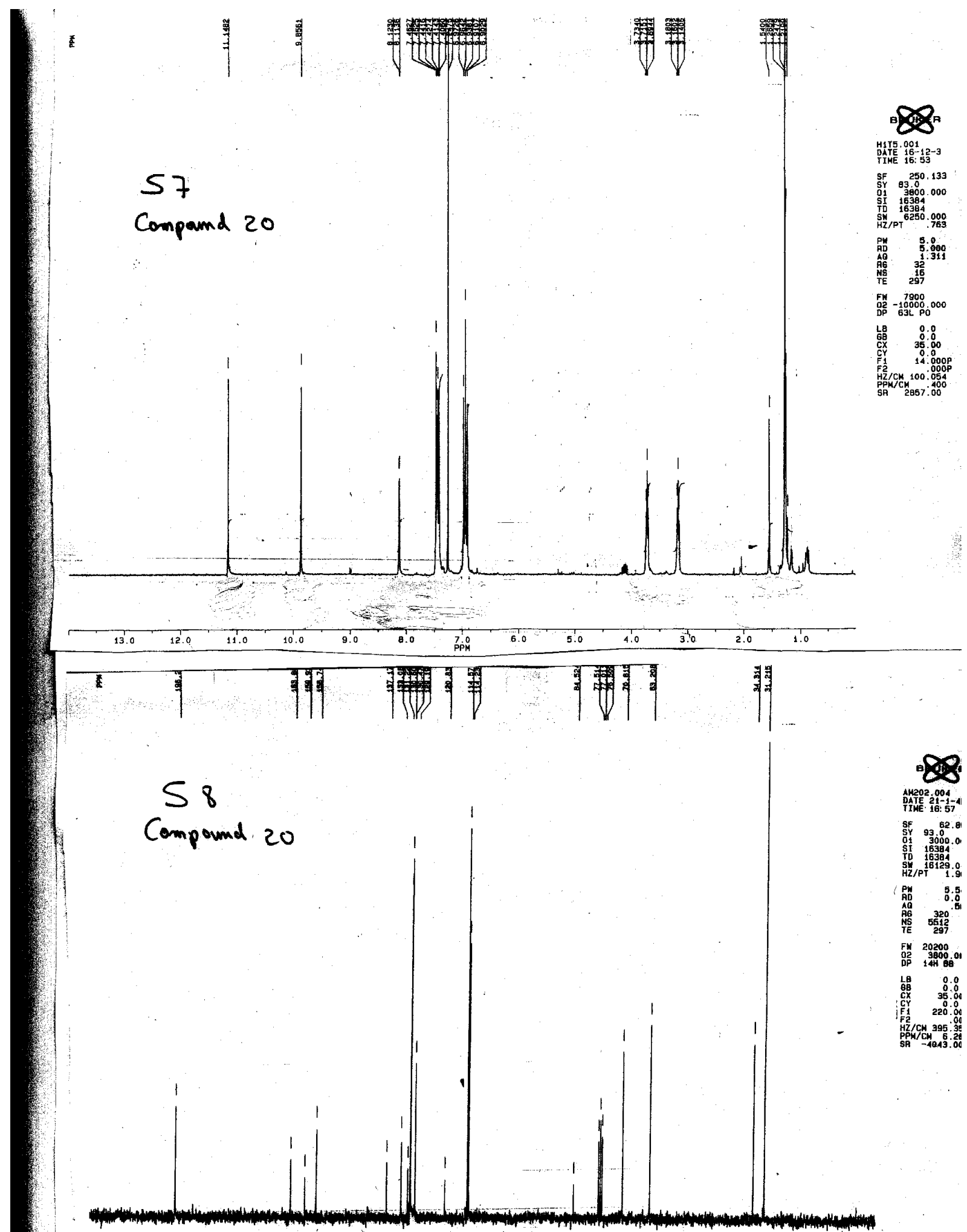

$\infty$

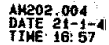

s5 93.82 .8

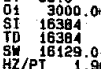

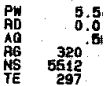

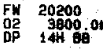

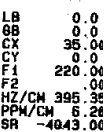

$200 \quad 100$

110

20 PPM 100

घ0

60

20 


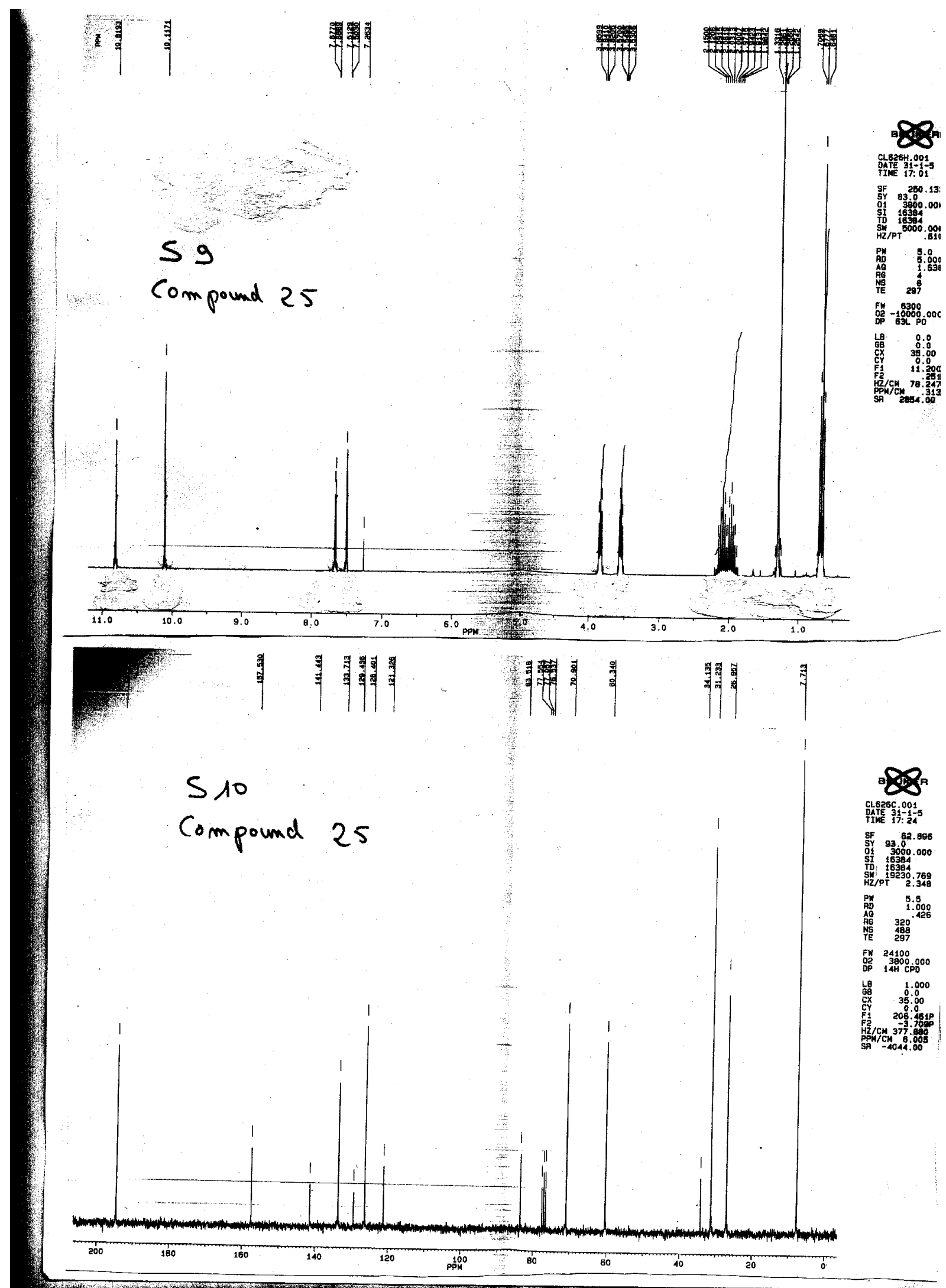




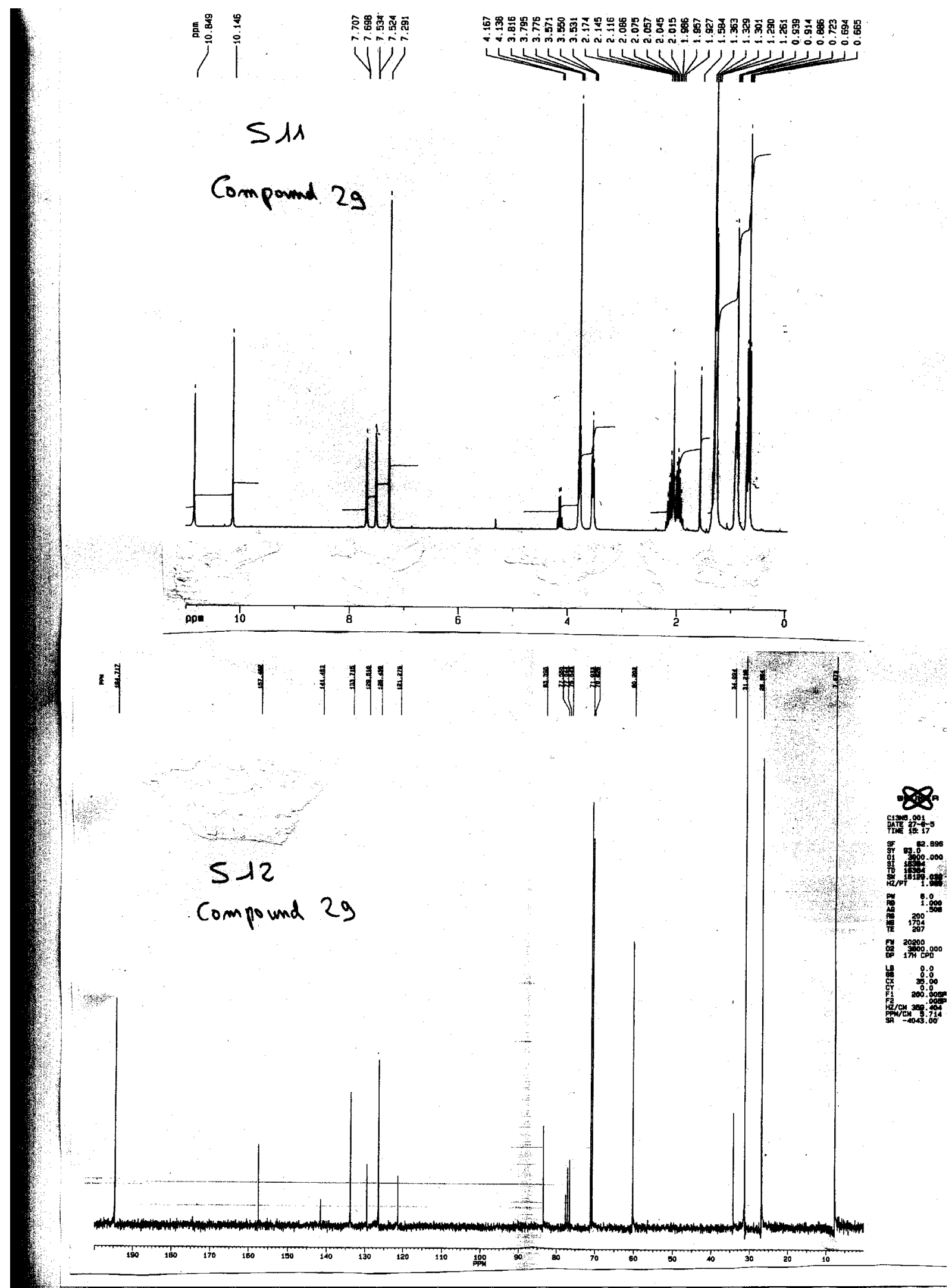

